\title{
Erratum to: An Entropy Functional for Riemann-Cartan Space-Times
}

\section{F. Hammad}

Published online: 22 September 2013

(C) Springer Science+Business Media New York 2013

\section{Erratum to: Int J Theor Phys (2012) 51:362-373 DOI 10.1007/s10773-011-0913-9}

The original version of this article unfortunately contained a mistake. The authors rectified the errors and are shown below:

We correct the entropy functional constructed in Int. J. Theor. Phys. 51:362 (2012). The 'on-shell' functional one obtains from this correct functional possesses a holographic structure without imposing any constraint on the spin-angular momentum tensor of matter, in contrast to the conclusion made in the above paper.

The error made in [1] was the missing torsion trace $Q_{i}=Q_{i j}{ }^{j}$ in the evaluation of the divergence terms. Indeed, the integral of a divergence in Riemann-Cartan space-times is of the form $\int_{M} \nabla_{i} V^{i}=\int_{\partial M} n_{i} V^{i}-\int_{M} 2 Q_{i} V^{i}$. Taking this into account, the entropy functional that should be considered is actually the following more 'economical' one

$$
\begin{aligned}
S= & \int \mathrm{d}^{4} x \sqrt{-g}\left[\alpha\left(\left(\nabla_{i} u^{j}\right)\left(\nabla_{j} u^{i}\right)-\left(\nabla_{i} u^{i}\right)^{2}\right)+\left(\lambda g_{i j}+T_{i j}\right) u^{i} u^{j}\right. \\
& \left.+\left(\Sigma_{i j k}+\Sigma_{i k j}+\Sigma_{k j i}\right) u^{i} \nabla^{j} u^{k}\right] .
\end{aligned}
$$

The additional terms introduced in [1] arise automatically when extracting total divergences. The variation of this functional with respect to the field $u^{i}$ reads

$$
\begin{aligned}
\delta S= & \int \mathrm{d}^{4} x \sqrt{-g}\left[2 \alpha\left(\left(\nabla_{i} \delta u^{j}\right)\left(\nabla_{j} u^{i}\right)-\left(\nabla_{i} \delta u^{i}\right)\left(\nabla_{j} u^{j}\right)\right)+2\left(\lambda g_{i j}+T_{i j}\right) \delta u^{i} u^{j}\right. \\
& \left.+\left(\Sigma_{i j k}+\Sigma_{i k j}+\Sigma_{k j i}\right) \delta u^{i} \nabla^{j} u^{k}+\left(\Sigma_{i j k}+\Sigma_{i k j}+\Sigma_{k j i}\right) u^{i} \nabla^{j} \delta u^{k}\right] .
\end{aligned}
$$

The online version of the original article can be found under doi:10.1007/s10773-011-0913-9.

F. Hammad $(\varangle)$

Département LMD-ST, Université A. Mira, Route Targa Ouzemmour Béjaia, W. Béjaia 06000, Algeria e-mail: fayhammad@gmail.com 
Extracting from the total divergences the boundary integrals which do not contribute to the variation, the condition $\delta S=0$ reads

$$
\begin{aligned}
& \int \mathrm{d}^{4} x \sqrt{-g}\left(2 \alpha \nabla_{[i} \nabla_{j]} u^{j}+\left(2 \alpha Q_{i} g_{j k}-2 \alpha Q_{k} g_{i j}+\Sigma_{i k j}\right) \nabla^{j} u^{k}\right. \\
& \left.+\left[\lambda g_{i j}+T_{i j}+\frac{1}{2}\left(\nabla^{k}+2 Q^{k}\right)\left(\Sigma_{i j k}+\Sigma_{k i j}+\Sigma_{k j i}\right)\right] u^{j}\right) \delta u^{i}=0 .
\end{aligned}
$$

From the identity $2 \nabla_{[i} \nabla_{j]} u^{j}=-R_{i j} u^{j}-2 Q_{i j}{ }^{k} \nabla_{k} u^{j}$, it follows that Eq. (3) is satisfied for arbitrary variations of $u^{i}$ if

$$
\begin{aligned}
& \left(2 \alpha Q_{k i j}+2 \alpha Q_{i} g_{j k}-2 \alpha Q_{k} g_{i j}+\Sigma_{i k j}\right) \nabla^{j} u^{k} \\
& \quad-\left[\alpha R_{i j}-\lambda g_{i j}-T_{i j}-\frac{1}{2}\left(\nabla^{k}+2 Q^{k}\right)\left(\Sigma_{i j k}+\Sigma_{k i j}+\Sigma_{k j i}\right)\right] u^{j}=0 .
\end{aligned}
$$

This in turn is satisfied for all $u^{i}$ if and only if the content of each of the two square brackets vanishes identically, leading straightforwardly as in [1] to the Cartan-Sciama-Kibble field equations, but containing more correctly than in [1] the metric $g_{i j}$ instead of the Kroneckerdelta $\delta_{i j}$ in front of the torsion traces $Q_{i} g_{j k}$ and $Q_{k} g_{i j}$. The arguments used in [1] to prove the uniqueness of the functional Eq. (1) remain unchanged. When the field equations are substituted in Eq. (1) after integration by parts, however, the 'on-shell' entropy functional becomes simply

$$
S=\frac{1}{8 \pi G} \int_{\partial M} \mathrm{~d}^{3} x \sqrt{|h|} n_{i}\left(u^{j} \nabla_{j} u^{i}-u^{i} \nabla_{j} u^{j}\right) .
$$

So contrary to the conclusion made in [1], the holographic structure emerges without imposing any constraint on the spin-angular momentum tensor of matter. Using Eq. (5), the application and conclusions made in [1] for the case of Dirac fields and black holes with intrinsic spin remain unchanged. For spin fluids obeying the Frenkel condition, however, the affine connection $\nabla$ in Eq. (5) reduces as in [1] to the Levi-Civita connection $\stackrel{\circ}{\nabla}$ only for an isotropic deformation $u^{i} u^{j}=\frac{1}{4} u^{2} g^{i j}$.

\section{References}

1. Hammad, F.: Int. J. Theor. Phys. 51, 362 (2012) 